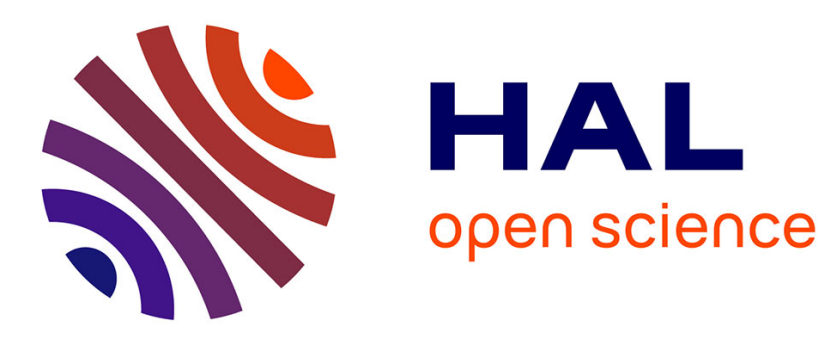

\title{
Time-resolved imaging using x-ray free electron lasers
}

Anton Barty

\section{To cite this version:}

Anton Barty. Time-resolved imaging using x-ray free electron lasers. Journal of Physics B: Atomic, Molecular and Optical Physics, 2010, 43 (19), pp.194014. 10.1088/0953-4075/43/19/194014 . hal00569847

\section{HAL Id: hal-00569847 https://hal.science/hal-00569847}

Submitted on 25 Feb 2011

HAL is a multi-disciplinary open access archive for the deposit and dissemination of scientific research documents, whether they are published or not. The documents may come from teaching and research institutions in France or abroad, or from public or private research centers.
L'archive ouverte pluridisciplinaire HAL, est destinée au dépôt et à la diffusion de documents scientifiques de niveau recherche, publiés ou non, émanant des établissements d'enseignement et de recherche français ou étrangers, des laboratoires publics ou privés. 


\title{
Time resolved imaging using X-ray Free Electron Lasers
}

\author{
Anton Barty ${ }^{1}$ \\ ${ }^{1}$ Centre for Free Electron Laser Science, Notkestraße 85, 22607, Hamburg, Germany.
}

(Dated: June 8, 2010)

\begin{abstract}
The ultra-intense, ultra-short X-ray pulses provided by X-ray Free Electron Laser (XFEL) sources are ideally suited to time resolved studies of structural dynamics with spatial resolution from nanometre to atomic length scales and temporal resolution of $10 \mathrm{fs}$ or less. Using coherent X-ray diffraction as a diagnostic, XFELs enable the capturing of X-ray snapshots of previously unmeasurable transient phenomena, and the study of ultrafast processes such as sample damage on the timescales which they occur. With enough photons in a single pulse to enable single-shot measurements, and short enough pulses to freeze atomic motion, researchers now have a new window into the time evolution ultrafast phenomena that are intrinsically not cyclic in nature. In this review paper we recap some of the key time-resolved imaging experiments performed at FLASH and look ahead to a new generation of experiments at higher resolution using a new generation of new XFEL sources that are only just becoming available.
\end{abstract}

\section{INTRODUCTION}

The unprecedented combination of high peak power, femtosecond pulses and penetrating X-ray radiation delivered by X-ray Free Electron Lasers offers researchers an entirely new window into previously unexplored regimes of the time evolution of matter. Capable of producing X-ray pulses ranging in length from less than 10 fs duration to more than 300 fs duration, and with up to $10^{13}$ monochromatic and spatially coherent X-ray photons per pulse, this new class of high peak power X-ray source is ideally suited to a new generation of time resolved studies.

At the same time new techniques in coherent X-ray imaging have opened up new frontiers in the imaging of condensed matter and biological samples on nanometre to atomic length scales free from the restrictions of fabricating high-quality X-ray imaging optics [55]. The combination of these two developments provide a powerful and unique new set of tools for the study of time evolution of matter on nanometre to atomic length scales, with temporal resolution approaching the timescales of atomic motion.

Understanding the dynamic response of materials under extreme conditions of pressure and temperature is a scientific quest that spans nearly a century. Critical to developing this understanding is the ability to probe the spatial and temporal evolution of the material structure and properties at the scale of the relevant physical phenomena. The effects of high powered X-ray and visible lasers on materials involve such processes as rapid ionisation, coulomb explosion, hydrodynamic expansion, spallation, ablation and non-thermal melting - phenomena that evolve on femtosecond to picosecond timescales and have intrinsic length scales ranging from nanometres to atomic length scales. Meanwhile current molecular dynamics simulations are able to predict the behaviour of materials up to a few microns in size based on the calculated motion and interaction of individual atoms. Experimental studies of material behaviour on length scales ranging from individual atomic motion to nanometre length scales are essential to bridging the gaps between model-based predictions and experimental reality.

In the field of biological imaging, theoretical calculations and numerical simulations predict that ultrashort and extremely bright coherent X-ray pulses will enable the diffraction patterns of large molecules, cells or other nanometre- to micron-sized objects to be measured before the sample explodes and turns into a plasma 8, 38, 54. Such predictions depend critically on the rate of sample damage, and careful experiments are required to validate these predictions. The relevant length scales for such investigations once again require simultaneous nanometre to atomic spatial resolution, and femtoseconds to picosecond temporal resolution of individual noncrystalline objects.

\section{A. The unique properties of XFEL sources for time resolved imaging}

X-ray free electron lasers are unique in providing extremely intense femtosecond pulses which deliver sufficient flux to capture images of isolated objects at nanometre to atomic resolution with a single shot. The photon energy provided by unseeded FEL sources currently lies in the X-ray regime of $100 \mathrm{eV}$ to $8 \mathrm{keV}$, enabling spatial resolution down to atomic length scales using X-ray diffraction techniques. X-ray pulse lengths are measured in femtoseconds providing ultrafast shutter speeds capable of freezing individual atomic motion. Pulses of less than $60 \mathrm{fs}$ are common, and pulses as short as 2 fs may be possible using very low emittance with reduced bunch charge 21. Using this technique sub $10 \mathrm{fs}$ pulses have been produced at LCLS however to date their duration has not been accurately measured. And with $10^{12}-10^{13}$ photons per pulse, each pulse contains a sufficient number of photons for performing individual measurements in a single-shot. Finally the almost complete transverse coherence and almost monochromatic spectral 
bandwidth enable the use of a wide range of newly developed coherent imaging techniques [55].

Alternative time-resolved imaging methods such as electron microscopes have long provided more than adequate spatial resolution for nanoscale studies, and have also been demonstrated with ultrafast pulses [74. However electron microscopes have limited penetrating power and struggle to obtain high quality single-shot images due to space-charge issues 3 . On the other hand optical laser pulses have made it possible to resolve dynamics on the femtosecond timescale, but the resolution achieved using optical techniques has been limited to a few micrometers 67.

Femtosecond X-ray pulses have been long been used to detect Ångstrom-scale atomic motions in extended crystalline materials with long-range order through timeresolved diffraction experiments [65, 66]. However, for the study of non-crystalline dynamics, higher peak power in short pulses are required than are available from conventional sources. X-ray beams from 3rd generation synchrotrons have comparatively long pulse lengths of 10-100 ps, as determined by the shortest electron bunch length possible in a given storage ring. Synchrotron sources can produce short pulse $(\approx 100 \mathrm{fs}) \mathrm{X}$-rays when operated as femtosecond slicing sources 62, but produce comparatively weak X-ray beams of $10^{7}$ photons/sec. Along with X-ray pulses from femtosecond laser plasma sources [57, 58 and high harmonic generation sources $[6$ this limits their use to non-destructive phenomena where weak signal can be accumulated over many repeatable excitations of the sample.

The unprecedented peak brightness and short pulses produced by X-ray free electron lasers provide the combination of penetrating power, spatial resolution and single-shot imaging capability necessary to probe a variety of transient phenomena at nanoscale to atomic length scales with simultaneous femtosecond temporal resolution. With up to $10^{13} \mathrm{X}$-ray photons per pulse [1] sufficient photons can be scattered from an isolated nanoscale object to perform coherent X-ray diffraction imaging using a single femtosecond-duration X-ray pulse [16, 18]. Meanwhile the 'shutter speed' of these measurements is ultimately determined by duration of the FEL pulse: for LCLS this is 60-200 fs (and less than 10fs in ultrashort puse mode [21]).

\section{B. Lensless imaging}

$\mathrm{X}$-ray imaging of isolated objects at nanometre to atomic resolution is necessarily lensless. The highest resolution achieved to date using zone plate lenses is in the soft X-ray regime where $15 \mathrm{~nm}$ spatial resolution has been demonstrated on a dense line-space periodic object [15. Meanwhile the high peak power produced by XFEL sources means that most optics - in particular high resolution zone plates - will not survive more than a single X-ray pulse. Atomic resolution is of course routinely
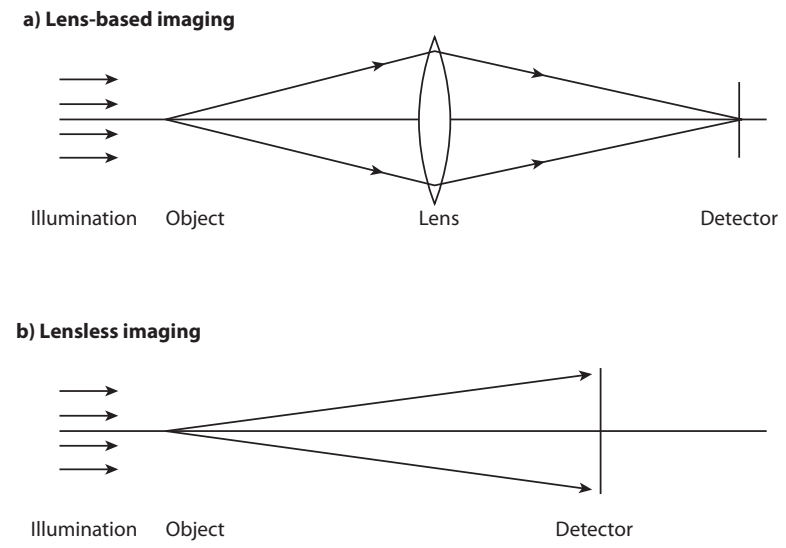

FIG. 1. Lensless imaging: The role of the lens in a conventional microscope (top) is to focus light scattered by the sample onto the detector plane through addition of an appropriate spherical phase to the scattered wave field. In lensless imaging (bottom) this process is replaced by a computational process: the intensity distribution is measured in the lens plane and converted into a real-space image using iterative phasing algorithms.

achieved on crystalline samples using X-ray diffraction, however the requirement for crystalline periodicity limits the range and type of samples that can be studied.

By directly measuring the far-field diffraction pattern of a non-crystalline (ie: non-periodic) object, lensless imaging can in theory deliver the atomic spatial resolution of X-ray diffraction measurements without the need for sample periodicity. Diffractive imaging replaces Xray lenses with computational lenses, Figure 1, reconstructing real-space images of the sample by applying phase retrieval algorithms to the properly sampled continuous far-field diffraction patterns produced by individual non-crystalline structures [22, 46, 48, 49, 51, 52. XFEL sources provide the high transverse coherence and spectral purity demanded by coherent diffractive imaging, whilst the use of detectors with a central hole avoids the challenges of designing beamstops capable of withstanding the intense XFEL pulses.

A few key concepts are pertinent to the discussion in this paper:

(1) The spatial resolution is determined by a combination of the photon wavelength and the range of diffraction angles measured on the detector, and is the same as the coherent resolution limit for a lens of the same size as the detector: $\Delta d=\frac{\lambda}{2 N A}$, where $N A$ is the numerical aperture of the imaging system $N A=\sin \theta$ and $\theta$ is the scattering angle at the edge of the detector [11. As with lens-based imaging systems the path to higher resolution (smaller $\Delta d$ ) is through either increasing the system numerical aperture $N A$ or decreasing the photon wavelength $\lambda$. Since $N A$ has an upper bound of 1.0 the main pathway to increased resolution is through the use 




b) Imageable area

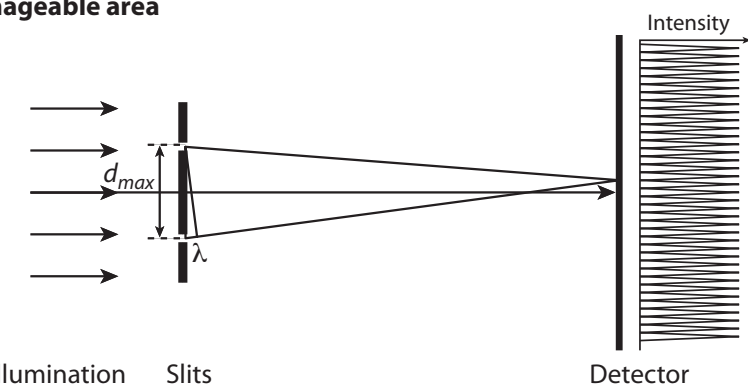

c) Temporal coherence limitation

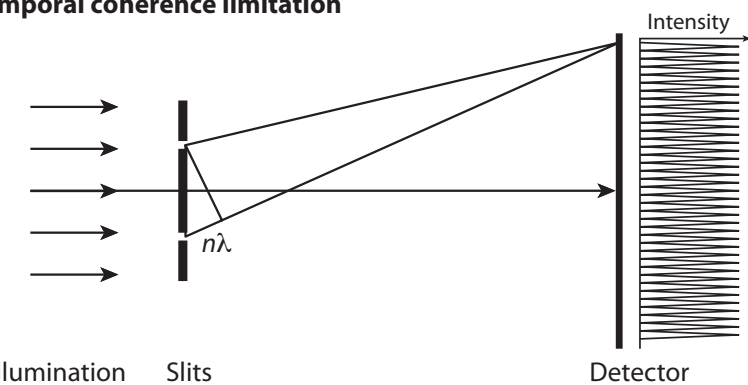

FIG. 2. Constraints in coherent imaging: (a) Spatial resolution is defined by the finest pitch grating that can be resolved and is determined by the wavelength of illumination and the numerical aperture subtended by the detector. (b) Image reconstruction requires the interference fringes from either side of the object to be adequately sampled by the detector: for objects above a certain lateral extent interference fringes in the detector plane will be aliased. The imageable area is determined by wavelength, detector distance and detector pixel size. (c) Temporal coherence of the FEL radiation also limits the extent of the object which can be imaged at a given resolution. Light scattered from both sides of the object must be temporally coherent in order to form interference fringes in the detector plane or fringe contrast is lost.

of shorter wavelength X-rays.

(2) The temporal resolution of a time resolved measurement depends on two things: the length of the probe pulse (the 'shutter speed') and the accuracy and repeatability of timing synchronisation between pump and probe pulses ('timing accuracy'). For single-shot coher- ent imaging using XFEL pulses as the probe the 'shutter speed' is given by the length of the X-ray probe pulse - tens of femtoseconds, or even less than $10 \mathrm{fs}$ in low charge mode. However the timing accuracy depends on the methods by which the pump pulse is synchronised and will vary depending on how each experiment is executed; see for example [14.

(3) For image reconstruction the speckle pattern formed on the detector must sampled according to the Nyquist sampling criterion or better. In the language of X-ray crystallography Nyquist sampling actually corresponds to oversampling of the Bragg spots formed on the detector (to crystallographers adequate sampling refers to measuring the intensity of each Bragg spot or bright peak) and this is the origin of the common term 'oversampled diffraction pattern' 60]. For image reconstruction it is therefore necessary to have sufficient pixels on the detector to properly sample each of the individual coherent speckles.

(4) The imageable area is the region on the sample over which image reconstruction is possible and is determined by a combination of many factors including the transverse and longitudinal coherence of the photon beam, detector properties, and the pulse length. Consider, for example, coherent interference from the two opposite sides of a finite sized object as measured in the detector plane. Firstly, the pulse length must be sufficiently long that the scattered waves from both sides of the object overlap in time in the detector plane. If the two scattered waves do not temporally overlap the result will be summation of intensities rather than coherent interference in the detector plane. Secondly, for an illuminating wave field with high transverse coherence - such as produced by an FEL - the imaged field is determined by the longitudinal (temporal) coherence of the photon beam required to achieve coherent interference at maximum resolution. Consider that the smallest resolved spatial period on the sample corresponds to one wavelength phase shift between the scattered waves from adjacent features when added coherently at the edge of the detector. If the longitudinal coherence length is some finite value, say $1000 \lambda$, then features separated by more than 1000 resolution elements no longer add coherently in the detector plane and the formation of coherent speckle is lost. Put another way, if the path length difference between two points on the sample to a given pixel on the detector is greater than the longitudinal coherence length one will get summation of intensities rather than interference between the two scattered waves from the sample. This in turn sets an upper bound on the size of the sample regions that can be probed using coherent scattering techniques. We describe the coherence limit separately from the pulse length limit because many XFEL sources are not transform limited, ie; their temporal coherence length is significantly less than the physical pulse length. Finally, for image reconstruction to work the pixel pitch on the detector must be sufficiently small to resolve the interference pattern produced by the object [60]. Consider, 
for example, the two-slit interference pattern projected onto the detector by two slits on opposite sides of a finite sized object: diffraction from these slits will produce an intensity modulation in the detector plane characteristic of two-slit interference. If the pixel pitch of the detector is not sufficiently fine to properly sample this intensity modulation the measured interference pattern will be aliased and the resultant image reconstruction will not be a true representation of the object. As the object gets larger, this two-slit interference pattern gets finer and more pixels are required to properly sample the interference pattern. Turning this argument around, for a given detector pixel pitch (pixels across the detector for a given spatial resolution) there is an upper bound to the physical extent of the object which can be imaged. If the sample is larger coherent scattering analysis can still be applied, but image reconstruction will not work. Thus if image reconstruction is desired the sample must be designed so that it is completely bounded within an area determined by the finest two-slit interference pattern than can be measured by the detector.

For further details a separate paper devoted to coherent X-ray imaging can be found later in this volume [1], and an excellent review of coherent X-ray imaging methods in general can be found in 55 .

\section{Scope of this review}

X-ray free electron lasers (XFELs) have only recently become available to researchers. The first XFEL to reach the soft X-ray regime, the FLASH facility in Hamburg, Germany, first became available as a user facility in 2005 . Meanwhile the first hard X-ray FEL, the LCLS facility in Stanford, California, opened its doors to users in October 2009.

In this review we explore some of the unique timeresolved science performed at FLASH since it opened to users and look forward to the range of future experiments enabled by hard X-ray FELs that are just becoming available to researchers worldwide.

\section{TIME RESOLVED IMAGING AT FLASH}

The FLASH facility in Hamburg was the first free electron laser to reach into the X-ray regime. Initially operating at wavelengths greater than $32 \mathrm{~nm}$ in 2005 and reaching down to $7 \mathrm{~nm}$ in 2009, this facility has been a proving ground for a range of ultrafast X-ray imaging techniques. Here we briefly review some of the key scientific discoveries made at FLASH using ultrafast X-ray imaging over the past 8 years.

\section{A. Outrunning radiation damage processes}

One of the first questions asked about the potential use of XFEL sources for imaging applications was whether it was possible to capture meaningful diffraction patterns from a sample before the sample itself was destroyed. Given the intense peak power provided by XFELs any material placed in the focal spot is turned into a plasma and destroyed. Even with modest focal spot sizes of 30 $\mu m$ diameter and pulses of only $3 \mu J$ in 25 fs the peak X-ray power reaches $4 \times 10^{13} \mathrm{Wcm}^{-1}$. A simple silicon nitride membrane placed in this focal spot is heated to over $60,000 \mathrm{~K}$ and destroyed.

The question to be answered was: will the sample be destroyed and turned into a plasma before any meaningful diffraction pattern can be recorded? The implications of such an experiment are significant as the use of ultrashort pulses might possibly provide a means for circumventing the traditional radiation damage limits in static $\mathrm{X}$-ray imaging 34.

Theoretical calculations and numerical simulations had already predicted that using an ultrashort and extremely bright coherent X-ray pulse, a single diffraction pattern could be recorded from a large molecule, cell or other nanometre- to micron-sized objects before the sample explodes and turns into a plasma [8, 38, 54. In normal Xray imaging experiments it is well known that radiationinduced damage and sample movement prevents the accumulation of high-resolution scattering from micron to nanometer sized objects 34 , 35. If diffraction data could be collected faster than the relevant damage processes, predictions were that the effects of radiation damage could be substantially reduced [54, 69]

The first experimental confirmation of these predictions came in the experiment of Chapman et.al [16 in 2006. In this experiment a simple test object - a microfabricated silicon nitride membrane - was placed in the focus of the FLASH beam. X-rays from a single FEL pulse diffracted from the sample and were collected using a novel X-ray camera [5, Figure 3. This camera employed a multilayer mirror to deflect X-rays scattered from the sample onto a conventional CCD detector located below the X-ray beam. This geometry is necessitated because any material in the X-ray beam is turned into a plasma - the central hole in the multilayer mirror acts as a convenient 'beamstop' obviating the need for a detector with a central hole.

A single-shot coherent diffraction pattern acquired from the sample is shown in Figure 4, along with diffraction from a second FEL pulse 20 seconds later. Calculations of the deposited energy indicate that the absorbed energy for the first pulse was approximately 20 $\mathrm{eV}$ per atom, heating the sample to a temperature of about $60,000 \mathrm{~K}$. The second diffraction pattern clearly shows different structure, and indicates that the sample had been destroyed by the first FEL pulse. Nevertheless, it is possible to reconstruct an image of the initial sample using one of any number iterative transform algorithms 


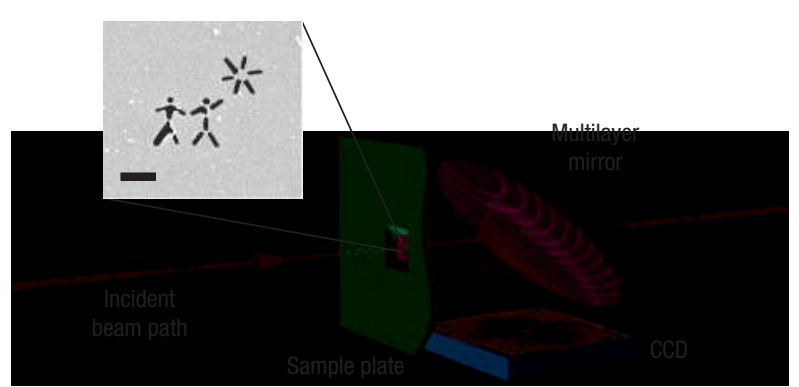

FIG. 3. Experimental arrangement for the first ultrafast X-ray diffraction image acquired at an FEL: The FEL beam is incident from the left and is focused to a $20 \mu \mathrm{m}$ spot on the sample, which consisted of a 20 -nm-thick transmissive silicon nitride membrane with a picture milled through its entire thickness using an focussed ion beam (FIB) (the sample is enlarged in the inset, and the scale bar indicates $1 \mu \mathrm{m})$. The direct beam passes through the sample window and exits the camera through a hole in a graded multilayer planar mirror. The diffracted light from the sample reflects from this mirror onto a CCD detector. The contour lines on the mirror depict lines of constant incidence angle (constant multilayer period). The on-axis path length from the sample to the detector is $55 \mathrm{~mm}$. For $32 \mathrm{~nm}$ radiation and objects smaller than $20 \mu \mathrm{m}$, this distance is in the far field, where the diffraction pattern is equal to the Fourier transform of the exit wave. The numerical aperture of the detector is 0.25 . Figure extracted from Chapman et.al, 2006.

22, 46, 48, 49, 51, 52. In this case the Shrinkwrap 48, algorithm was used because it required very little $a$ priori knowledge of the same structure other than a well-defined upper bound on the area or volume of the "imaged field" that can be occupied by the sample. Analysis of the reconstructed image indicates that the image is diffraction limited by the detector solid angle: the image shows no effects of radiation damage to the sample. This $t=0$ experiment effectively demonstrates that that radiation damage processes in this sample at the $15 \mathrm{~nm}$ spatial scale occur on time scales longer than the FEL pulse duration of $25 \mathrm{fs}$.

This ground-breaking experiment was critical in demonstrating that XFEL pulses could outrun sample damage and collect meaningful diffraction patterns encoding sample structure to diffraction limits before the sample itself was destroyed. Variations on the principle of flash X-ray imaging demonstrated in this experiment have subsequently been used in a wide range of coherent experiments at FLASH and elsewhere [7, 9, 12, 18, 19, 33, 50.

\section{B. Single-shot imaging of particles in flight}

The single-shot imaging capabilities demonstrated by Chapman et.al. in 2006 have been exploited by Bogan et.al 9 to study the morphology of aerosols in free flight, Figure 5 These studies revealed the shape and structure
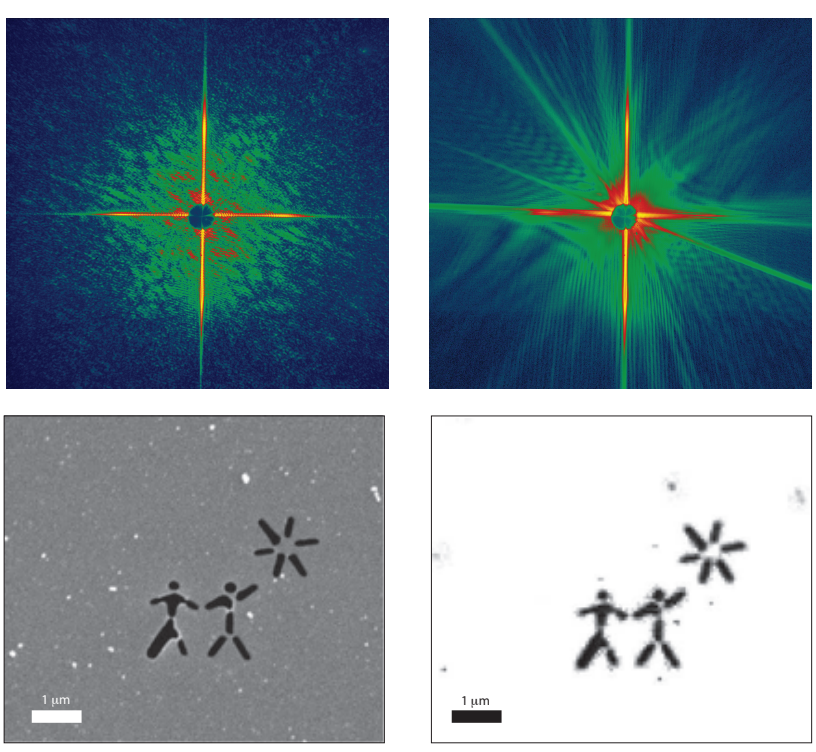

FIG. 4. Femtosecond X-ray diffraction outruns sample damage: Top: Coherent diffraction patterns recorded for a single $(4 \pm 2) \times 10^{14} \mathrm{Wcm}^{-2}, 25 \pm 5$ fs pulse (left) and for the subsequent pulse of similar intensity and duration, $20 \mathrm{fs}$ later (right) showing diffraction from the damage caused by the initial pulse. Bottom: SEM image of the sample before exposure to the FEL beam (left), and image reconstructed, from the ultrafast coherent diffraction pattern (right). Composite figure extracted from Chapman et.al, 2006.

of micron-sized particulate matter in free space without the need to suspend the particles on a supporting membrane - a significant advance over previous techniques that required samples to be first attached to a membrane for study by electron microscopy. Since the free-flying particles are moving at speeds of several hundred meters per second traditional electron microscopy techniques are not suitable - an alternative imaging technique such as flash imaging that offers both nanoscale-resolution and a fast enough shutter speed to freeze sample motion is required. This topic is the subject of a completely separate paper in this volume and more details can be found in [10.

Similar experiment have been performed on individual cells in free flight, however to date the only cell imaging papers published in the literature are of cells on fixed membranes. In 2008 Marchesini et.al. 50 used single-shot holography to image cells deposited on membranes with pre-prepared holographic reference structures, whilst in 2010 Mancuso et.al. [47] exploited the high spatial coherence of FLASH to image cells fixed on membranes in an experiment similar to coherent imaging techniques employed in synchrotron experiments 36, 42, 64 by accumulating signal from the same sample over many weak pulses without each individual shot destroying the sample . 


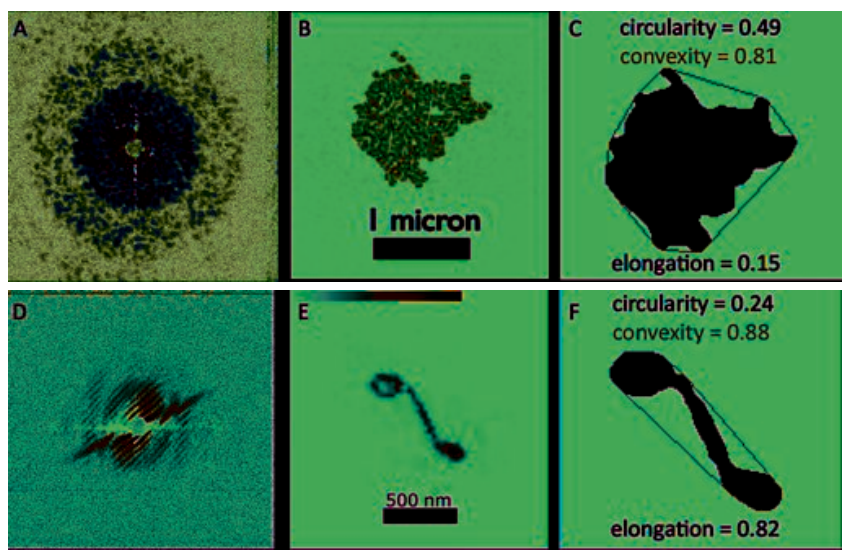

FIG. 5. Fast single-shot imaging of aerosols in flight: Micron-sized aerosol particulate matter images using singleshot diffractive imaging by Bogan et.al 9. Phase retrieval applied to coherent diffraction patterns from gold nanoclusters (A) and carbon fibers (D) yields real-space images of the particulate morphology (B and E) and from this important information about the shape of the particulates in free flight ( $\mathrm{C}$ and $\mathrm{F}$ ). The free-flying particles are moving at speeds of several hundred meters per second necessitating the use of a fast imaging technique with nanoscale resolution to freeze the sample motion. Figure extracted from Bogan et.al. 2010.

\section{Measuring the speed of sample damage}

The first flash X-ray imaging experiment [16] demonstrated that femtosecond pulses from XFEL sources could collect meaningful diffraction patterns from samples before they were destroyed. However, since no damage to the sample was observed the question of measuring the rate at which the sample was being damaged remained. To answer this question a time-resolved experiment was required, and the experiment had to use an X-ray pump to drive the sample.

Ultrafast time-resolved methods require a high degree of synchronization between pump and probe pulses to initiate a transition and then probe it after a precisely defined time delay. In practice this is difficult to achieve in X-ray pump-probe experiments due to the challenges involved in producing split-and-delay X-ray optics. In another groundbreaking experiment, Chapman et.al. 18. employed a novel scheme of time resolved holography to probe sample evolution after a well defined time delay. To do this the sample is placed near an X-ray mirror; after the pulse traverses the sample triggering the reaction, a multilayer mirror reflects the pulse back onto the sample to probe the reaction. This creates a compact X-ray pump-probe geometry from a single FEL pulse requiring only a single normal-incidence multilayer-coated mirror, in which the time delay is determined by the time taken for light to travel from the sample to the mirror and back. Commonality of path lengths between the pump and probe beams ensures temporal coherence between the two pulses leading to interference rings in
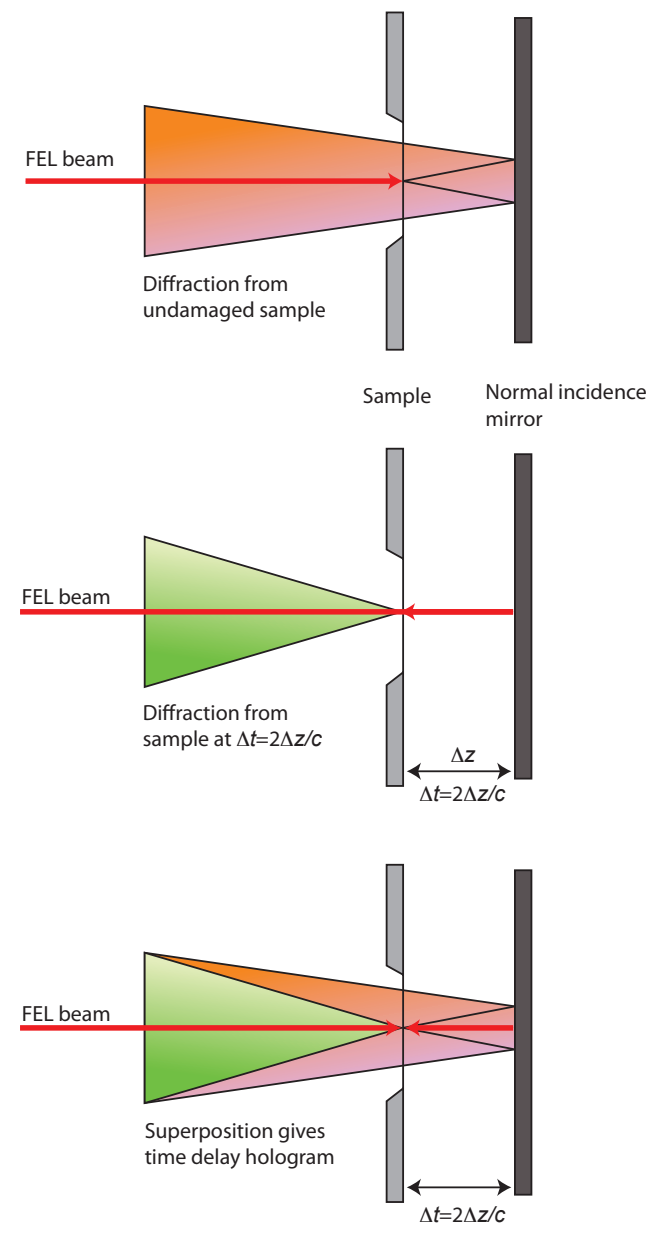

FIG. 6. Geometry for time-delay holography The incident FEL pulse from the left is incident on the sample, which is in turn backed by a multilayer-coated plane mirror. The mirror returns the direct beam back through the the sample a second time at a time delay given by the distance travelled from sample to mirror and back. The prompt diffraction (orange, the reference wave) and delayed diffraction (green, the object wave) interfere to generate the hologram on the detector encoding both time delay and evolution of the sample structure.

the measured image. Structural changes in the sample are holographically recorded in the structure of the measured diffraction patterns, and the time delay is simultaneously encoded in the ring spacing of the diffraction pattern. This ring structure encodes the time delay to an accuracy of one femtosecond.

The layout for time-delay holography is shown in Figure 6. The FEL beam, incident from the left, diffracts from the sample in the same way as in a conventional diffraction imaging experiment (top). Both the diffracted and undiffracted X-rays are reflected from a normal incidence multilayer mirror placed downstream of the sample back towards the FEL source, striking the sample for a second time (middle). The FEL beam then diffracts from 


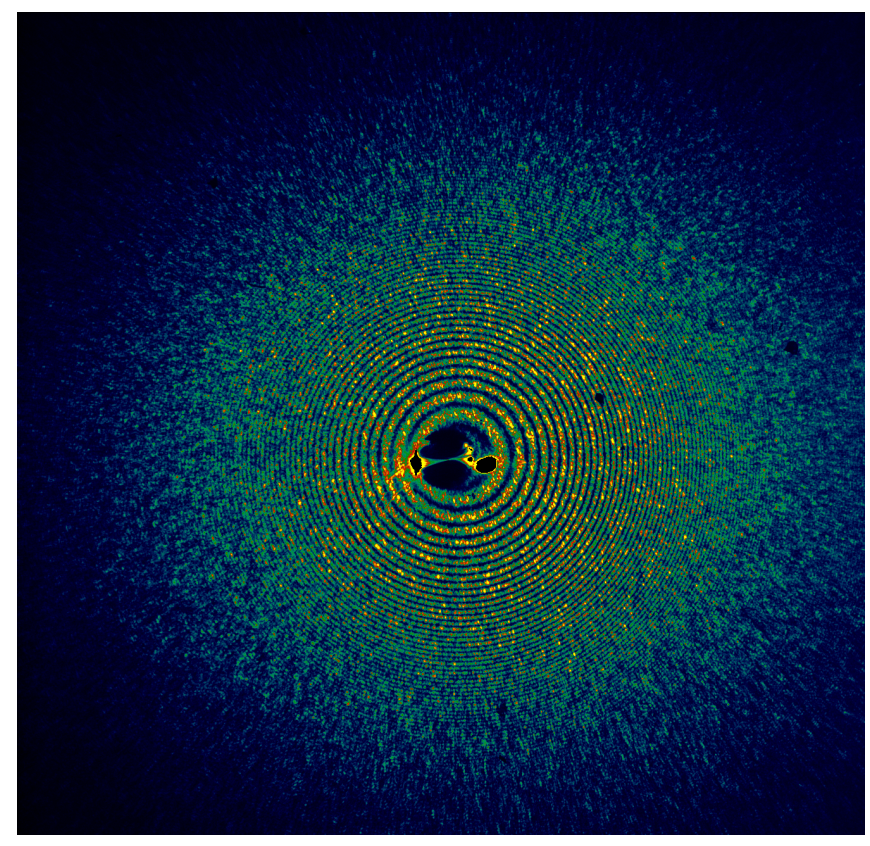

FIG. 7. Time-delay hologram recorded at FLASH Coherent speckle in the detector plane is formed by the inferference between the sample initial and time-delayed diffraction patterns, whilst the Newton's rings formed in the detector plane encode the time delay to femtosecond accuracy. Colour version of figure extracted from Chapman et.al. 2007.

the sample a second time (middle) after a time delay determined by time of flight from the sample to the mirror and back. Because both pump and probe beams have travelled the same distance they interfere in the detector plane (bottom). Coherent speckle in the detector plane is formed by the inferference between the sample initial and time-delayed diffraction patterns, Figure 7, whilst the time delay is encoded in the 'Newton's rings' formed in the detector plane to femtosecond time delay accuracy. In practice this experiment was realised using a multilayer mirror camera similar to that shown in Figure 3 rotated $180^{\circ}$ so that the XFEL was incident from behind the multilayer mirror [18]. This time-resolved holographic setup was used to study the dynamics of materials in the extreme conditions of intense FEL pulses, both during the pulse and as it turns into a plasma.

The point of this experiment was to measure the rate of sample damage, and thereby understand both fundamental light-matter interactions [41] and the ultimate resolution limits of flash imaging techniques [18. A sample loaded with spherical polystyrene particles was used as the test object. 32.5-nm-wavelength pulses from FLASH with individual pulse energies between $2 \mathrm{~mJ}$ and $18 \mathrm{~mJ}$, focussed to a $20-\mu \mathrm{m}$ gaussian-profiled spot, produced peak intensities of $10^{14} \mathrm{Wcm}^{-2}$ on the sample peak intensities corresponding to more than 5,000 times the damage threshold of the polystyrene particle [28]. Polystyrene spheres were chosen as the sample because spherical particles are the simplest shape to consider for modeling X-ray matter interaction dynamics, and polystyrene spheres are both uniform in composition and can be size-selected to a well defined size distribution to give accurate scattering measurements.

It was observed that the hologram intensity envelope is unchanged for delays shorter than $1 \mathrm{ps}$, becoming narrower with longer delays, indicating that the particle diameter begins to increase. This narrowing of the structure factor is observed for delays longer than $3.8 \mathrm{ps}$, in agreement with simulations that predict the transverse particle diameter has increased by $40 \%(60 \mathrm{~nm})$ at 3.8 ps. A sample expansion of only $(6 \pm 3) \mathrm{nm}$ at $350 \mathrm{fs}$ delay was estimated from the data to be an upper bound of the explosion. Extrapolation of these measurements to the FEL pulse duration of $30 \mathrm{fs}$ predicts an expansion of no more than $0.4 \mathrm{~nm}$ had occurred during the length of the FEL pulse. Based on these observations of sample explosions in the FEL beam it appears feasible to overcome conventional radiation damage limits in soft$\mathrm{X}$-ray microscopy of cells [35] using sufficiently short and intense X-ray FEL pulses [69.

\section{Slowing the sample damage rate}

As the resolution demanded of ultrafast single-shot imaging experiments progresses towards atomic or nearatomic resolution the achievable resolution of images is likely to depend as much on the degree of radiation damage to the molecule that occurs during the course of the $\mathrm{x}$-ray pulse as on signal to noise in the image. Indeed as samples become smaller the scattering signal becomes weaker necessitating more intense pulses to maintain signal to noise. At the same time, more intense pulses lead to more rapid degradation of the sample as correspondingly higher X-ray fluxes are required. Finding the optimum trade-off between maintaining signal to noise through higher incident flux levels whilst limiting sample damage to an acceptable level is critical. Any technique that can retard the rate of sample damage long enough for high-resolution images to be captured is extremely valuable. Such a technique would enable either an increase in delivered flux and therefore signal to noise in the detected diffraction pattern, or a relaxation of the FEL pulse length required to achieve a given spatial resolution.

An experiment performed at FLASH by Hau-Riege and co-workers [33] showed that sample explosion could be retarding by coating the sample in carefully selected optically opaque sacrificial layers. Commonly called tamper layers (after their use in the Manhattan project to retard very large explosions) such sacrificial layers are optically transparent, yet arrest hydrodynamic expansion through inertial confinement whilst supplying a bath of photo-induced free electrons to the sample quenching coulomb explosion of the sample itself. Hydrodynamic simulations suggest that without a tamper, the sample disintegrates on the time scale of a few picoseconds 

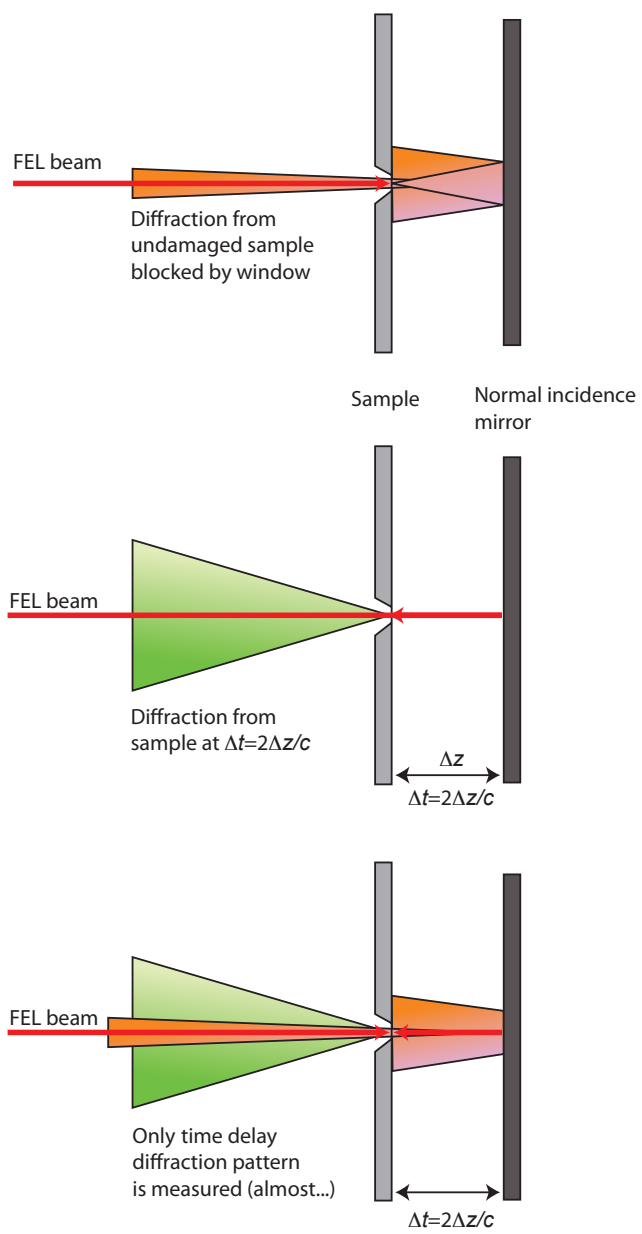

FIG. 8. Imaging in a time-delay holography geometry Time-delayed imaging can be performed in the holographic geometry if the reference wave is blocked. Here, the FEL pulse is once again incident from the left. After interaction with the sample both the initial diffraction and direct beam are reflected from a normal-incidence multilayer-coated plane mirror. The mirror returns the direct beam back through the the sample a second time at a time delay given by the distance travelled from sample to mirror and back, where the direct FEL beam once again diffracts from the sample. Small windows surrounding the sample block the prompt diffraction (orange, the reference wave), leaving only the time-delayed diffraction (green, the delayed diffraction) incident on the detector. Normal phase retrieval techniques can then be applied to image the time-delayed sample structure.

whereas with a tamper the sample more-or-less retains its shape 33 .

An elegant variant of the time delay holography technique was used to measure the effect of a tamper on sample damage rates, Figure 8. By mounting the sample on very small windows the primary diffracted light is prevented from reaching the detector after it is reflected back from the mirror; in particular if the reflecting mirror is located sufficiently far away from the sample only diffraction from the time-delayed sample is measured on the detector. In such cases the detected signal looks just like a normal speckle pattern measured in conventional diffraction imaging experiments and can be readily reconstructed using iterative phasing techniques described in detail elsewhere. For small time delays the normal incidence mirror is close to the sample and some of the initial diffraction can pass through the windows, interfering to produce Newton's rings and calibrating the mirror position. Translation of the mirror on calibrated stages then enables absolute knowledge of time delays even when the prompt diffraction is blocked. Using small window sizes has the further advantages that the data analysis is significantly simplified and that the overlap of the pump and probe beam is ensured.

Although atomic resolution imaging experiments are not possible in this geometry at FLASH due to the comparatively long X-ray wavelengths, the sample for this experiment was was selected to closely mimic the case of a hard X-ray FEL imaging experiment of molecules embedded in a tamper film, but on length scales that are accessible to the $13.5 \mathrm{~nm}$ wavelength available at FLASH at the time of these experiments. The sample consisted of cylindrical aluminum pillars deposited on $100 \mathrm{~nm}$-thick silicon-nitride membranes, spanning $7.5 \mu \mathrm{m}$ square windows etched into a silicon wafer [see inset in Fig. 3(c)]. An SEM image of the aluminium pillars is shown in the insert to Figure 9 . Some of the membranes were coated with a $100 \mathrm{~nm}$-thick silicon tamper layer which is optically transparent at the probe wavelength $(13.5 \mathrm{~nm})$. For this experiment the X-ray wavelength was $13.5 \mathrm{~nm}$, the pulse length $25 \mathrm{fs}$, and the fluence $31 \mathrm{Jcm}^{-2}$. Hydrodynamic simulations indicate that the physics describing the macroscopic motion of spherical and planar samples are equivalent.

Only coherent diffraction from the returning probe pulse is collected on the detector, simplifying interpretation of the time-delay diffraction patterns. For this sample any expansion of the aluminium pillars causes a change in the Airy-like envelope of the diffraction pattern, whilst the relative positions of the pillars is encoded in the coherent speckle pattern, as shown in Figure 9. The difference between tampered and untampered sample is immediately apparent: Without a tamper layer, (a), the diffraction minima have shifted to significantly smaller angles, indicating that the aluminum pillars have expanded. However with a tamper layer present, (b), the diffraction patterns of the low- and high-fluence exposures are similar and the diffraction Airy-like minima are at similar positions.

Since the measured image is simply a coherent diffraction pattern from the time delayed sample, conventional phase retrieval techniques can therefore be used to calculate images of the sample at the various time delays, enabling imageing of the real-space sample evolution over time. This imaging of sample expansion is shown in Figure 9(c). Here data collected using many low-fluence pulses captures the undisturbed sample structure, whilst 
(a) Explosion of naked sample

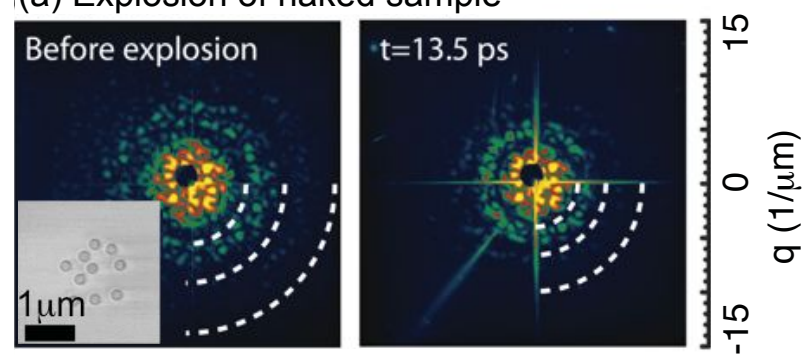

(b) Retarded explosion of tampered sample

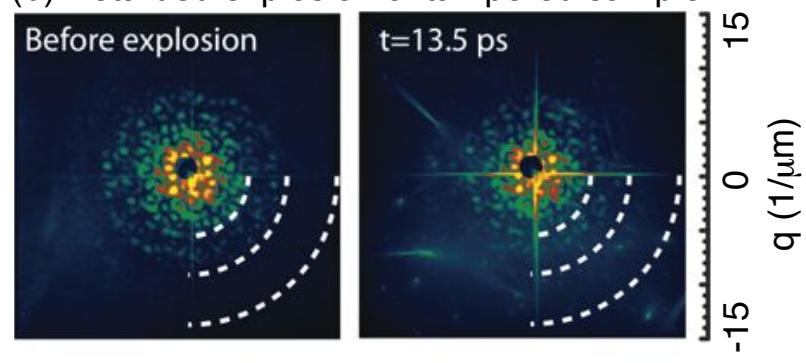

(c) Difference between unexploded and $\mathrm{t}=13.5 \mathrm{ps}$ images

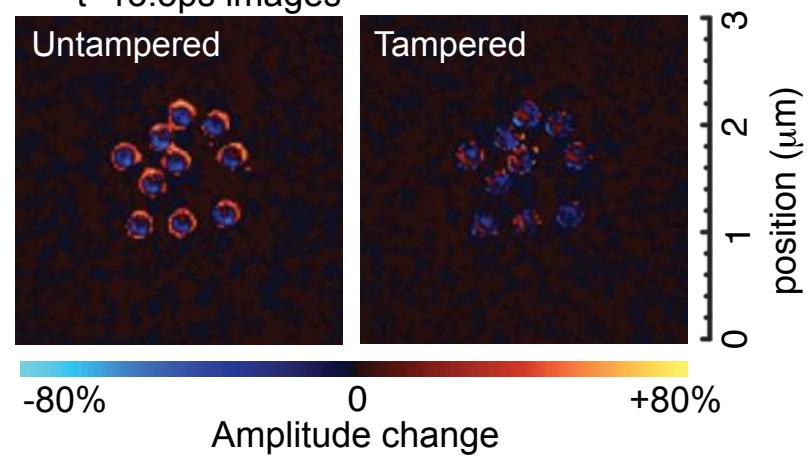

FIG. 9. Retardation of sample explosion as measured using small-window time-delay holography: Far-field diffraction patterns from (a) the naked, untampered sample and (b) from the tampered sample both before explosion and 13.5 ps after the drive pulse. Arcs highlight Airy ring minima in the measured diffraction pattern. Contraction of the arc radius indicates expansion of the sample, and it can be clearly seen that sample expansion at the 13.5 ps time step is more significant for the untampered sample (a) than for the tampered sample (b). Image reconstruction was also performed using iterative phasing techniques and difference images for the two time steps are shown in (c). Light colors indicate significant density changes. The untampered sample (left) shows large expansion, whereas the tampered sample (right) shows minimal structural changes. Figure extracted from Hau-Riege et.al. 2010.

data from a single high-power XFEL pulse captures the sample explosion in progress. The difference between the two images shows the extent of sample explosion at each time delay, in this case 13.5 ps. Here, light colors indicate significant density changes as shown in the scale bar. It is immediately clear that the untampered sample (left) shows large expansion, whereas the tampered sample (right) shows minimal structural changes. This experiment demonstrates that a tamper layer ensures the integrity of the sample for a period of at least $5 \mathrm{ps}$ duration following irradiation with a 25 fs-long $13.5 \mathrm{~nm}$ FEL pulse, which is $200 \times$ longer than the pulse duration. From a technique perspective, the X-ray pumpprobe technique used in this experiment is currently the only method that enables single-shot imaging of the sample during the course of the explosion at a precise time points after x-ray excitation.

This approach to containing sample explosion presented in [33] is based a combination of the principle of supplying electrons to neutralize an ionized core combined with various degrees of inertial confinement as a means of delaying sample explosion. Hau-Riege et.al. use the results from this experiment to extrapolate to the case of higher-resolution molecular imaging. After 10 ps, the expansion of the aluminum pillars was observed to be less than $10 \mathrm{~nm}$, so that simple linear scaling to a pulse length of 25 fs may suggest that a tamper restrains motion to about $1 \AA$ during a 25 fs pulse. By contrast a similar scaling of motion for the untampered sample leads to motions much larger than demanded to achieve atomic resolution. Detailed discussion of the plasma physics involved in this extrapolation is beyond the scope of this review and further details can be found in Hau-Riege et.al. [33] and supporting material. A likely tamper candidate for biomolecules is water, already used in cryo electron microscopy in the form of vitreous ice. Extrapolation from the results of this experiment to future biological imaging experiments suggest that use of a sacrificial water tamper may efficiently delay hydrodynamic expansion of a $50 \AA$ diameter macromolecule during the course of a 25 fs X-ray pulse, which is a typical pulse length expected on upcoming hard x-ray FELs 33.

\section{E. Tracking the evolution of extreme non-equilibrium states}

Short pulse excitation by high powered lasers is capable of driving materials beyond fundamental stability limits into extreme non-equilibrium states, often resulting in permanent changes to the morphology of the irradiated surface and to the formation and ejection of nano-particles. Understanding such processes is of major importance for many technological applications such as laser machining, laser surgery, pulsed-laser-deposition, and nano-particle synthesis. It also of great interest from a fundamental physics point of view because important questions in phase transition physics are addressed.

When materials are driven beyond conventional stability limits fundamental changes in state occur, many of them transient and therefore not observable in steadystate experiments. Depending on the excitation strength and the material properties, the initial solid phase is turned into a disordered liquid, an over-critical fluid or directly into a dense plasma on sub-picosecond to pi- 
cosecond time-scales. Following an initial heating-anddisordering phase it is believed that the rapid adiabatic expansion of the pressurized material can drive the material into yet unexplored meta-stable or even unstable regions of the state diagram. The response of materials to extreme excitation is extremely complex and most of our understanding about the detailed behaviour of the various physical processes involves comes from the results of theory and simulations 45, 70, 75]. Designing diagnostics to clearly distinguish between one theory and the other is difficult. Many experiments have been performed to probe these states as best as possible [26, 27, 43, 44, 67], however direct experimental evidence in support of one model or another is still missing. The comparatively wide variation in results depending on experimental conditions and individual material properties only complicates matters further.

Critical to developing this understanding is the ability to probe the spatial and temporal evolution of the material structure and properties at the scale of the relevant physical phenomena - covering sub-nanometer to micrometer length scales and sub-picosecond to nanosecond time scales. While experimental investigations combining this range of spatial and temporal scales were unimaginable just a decade ago, the advent of X-ray free electron lasers, and in particular single-shot coherent imaging techniques, have brought this goal within reach for the first time. At the same time improvements in computational models and increases in computing power have seen large-scale molecular dynamics MD simulations performed on sufficient volumes to approximate bulk processes. These simulations predict the development of inhomogeneous structures with typical length scales of about 1-10 nm after ultrafast irradiation.

By combining the newly available single-shot imaging techniques using X-ray free electron lasers with highpower laser drive pulses on well characterised fixed targets it is now possible to perform experiments at spatial and temporal scales overlapping with the capabilities of large-scale molecular dynamics simulations. In this regard the superior beam quality available from FEL sources compared to, say, laser plasma backlighters, provides significantly better data. In particular the high spatial coherence available from FELs enables the use of coherent scattering techniques and, in combination with carefully selected samples, even coherent imaging techniques. The latter enables spatially resolved imaging with high resolution of the disintegration or phase change of a target, in contrast for example to the observation of a disappearing diffraction line which only provides information that crystalline structure is lost but does not give spatial resolution. Even without image reconstruction it is possible to calculate the time evolution of predicted X-ray speckle patterns from molecular dynamics models and compare this to data obtained in a real experiment. This provides a reciprocal space diagnostic on the characteristic statistical length scales involved in the process. If the sample is such that it is possible to reconstruct

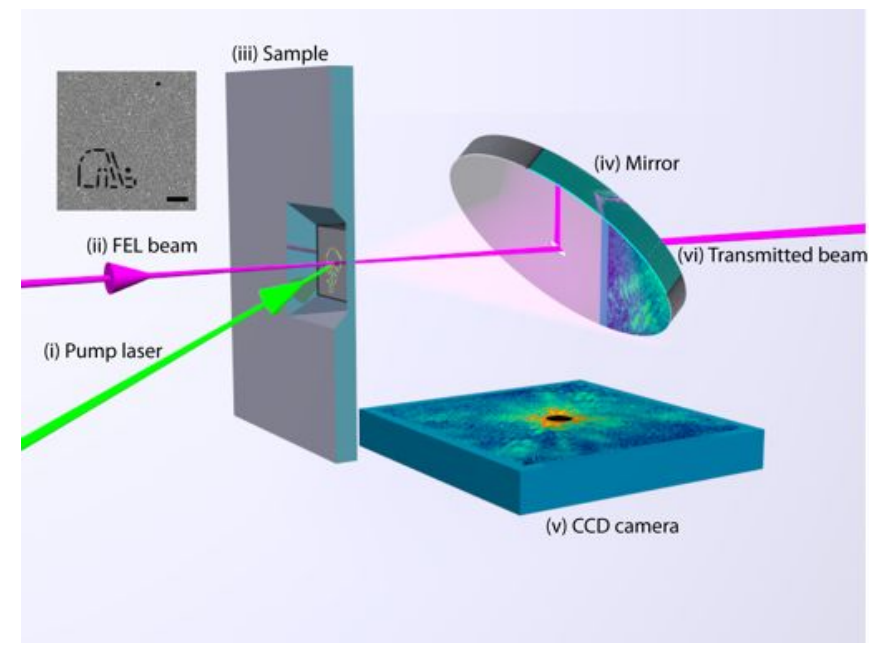

FIG. 10. Experimental arrangement for time-resolved diffraction imaging at FLASH: A visible light laser beam is focused onto the sample and acts as the excitation pulse. A $10 \mathrm{fs}$ duration soft X-ray pulse is focussed in the same location as the visible light laser and acts as the probe, diffracting from the sample and carrying information about the transient sample structure to the detector in the form of a coherent diffraction pattern. For the experiments at FLASH a $45^{\circ}$ mirror is used to separate the direct beam from the diffracted light, and a $100 \mathrm{~nm}$ thick zirconium filter over the CCD chip makes the detector blind to the laser excitation pulse. The spatial resolution is determined by the X-ray wavelength and the detected numerical aperture. Figure extracted from Barty et.al. 2008.

real-space images from experimental data, the electron density maps can be directly compared to the real-space models produced by molecular dynamics simulations. Finally, the femtosecond shutter speed available from FEL sources means that sample motion is properly 'frozen' in time, whilst the spatial resolution available using penetrating X-rays and characteristic sample size used in coherent imaging experiments is well matched to the length scales achievable with current MD simulations.

A significant step towards being able to visualise ablation processes in action was made by Barty et.al. in 2008 7]. Using a combination of coherent diffraction imaging, the femtosecond X-ray pulses produced by FLASH, and a synchronised laser drive pulse time-series snapshots of the strongly driven material was captured as it evolved on the ultrafast timescale.

The experimental arrangement for is shown in Fig. 10. A short-pulse linearly polarized laser (Nd:YLF operated at $523 \mathrm{~nm}$ ) was used to initiate material ablation in a structured silicon membrane sample. The drive laser pulse length was $12.5 \mathrm{ps}$ in duration and delivered $25 \mathrm{~mJ}$ per pulse into a $30 \mu \mathrm{m}$ FWHM focal spot on the sample, giving peak peak drive intensities of about $2.2 \times 10^{11} W_{c^{-2}}-$ well above the sample damage threshold. Femtosecond X-ray pulses from FLASH were used to probe sample evolution. Each pulse was $10 \mathrm{fs}$ in duration at $13.5 \mathrm{~nm}$ wavelength focused to a $20 \mu \mathrm{m}$ FWHM 
spot on the sample. Spatial overlap between the beams was achieved using a long working distance microscope, and a $0.25 \mathrm{NA}$ detector gave $27 \mathrm{~nm}$ spatial resolution in the sample plane. The mean energy of the X-ray pulses was $20 \mu \mathrm{J}$, resulting in $1.4 \times 10^{12} \mathrm{X}$-ray photons per pulse - sufficient to perform single shot diffraction imaging. Of course each FEL pulse destroys the sample as it is imaged. Independent measurements must therefore be carried out over a statistically significant number of separate measurements, creating an ensemble of individual snapshots that reveal both the mean and statistical variation of the physical processes involved.

Time-evolution of the sample at different delays after pump laser illumination is shown in the X-ray diffraction patterns of Figure 11, which span several picoseconds after excitation. The progressive loss of structure information at high scattering angles is immediately evident, corresponding to loss of mesoscale order as the sample disintegrates. Furthermore, new structure introduced into the sample by the ablation pulse is also clearly visible. The evolution of this structure gives rise to an increase in the intensity of fine speckles at spatial frequencies $q$ above about $5 \mathrm{\mu m}^{-1}$ primarily in the direction perpendicular to the pump laser polarization. Additionally a pair of strong diffraction peaks are clearly visible at $140 \mathrm{ps}$ delay. These structures are indicative of a light-induced periodic structure induced in the foil by the drive laser [3]. Also visible in the diffraction patterns is an increase in speckle intensity at lower frequencies at longer delays, corresponding to further loss of high spatial frequency order in the sample as voids in the sample formed during ablation merge and coalesce over time.

The coherent diffraction snapshots captured by each XFEL pulse are amenable to a range of analysis techniques, and analysis techniques such as time-resolved small angle X-ray scattering (SAXS) and time-resolved photon correlation spectroscopy (XPCS) can be applied to this type of data. For example computing the pixelwise cross-correlation between the initial state, Figure 11 (a) and subsequent time steps results in the $q$ dependent cross-correlation plots shown in Figure 12 . The speed at which order is lot can be measured and shows that disorder in the sample initially propagates at between 5,000 and $6,000 \mathrm{~ms}^{-1}$ during the first $20 \mathrm{ps}$, slowing to $1,000-2,000 \mathrm{~ms}^{-1}$ by $\mathrm{t}=140 \mathrm{ps}$ as the resultant plasma expands and cools. . This propagation of disorder corresponds to speed of the explosion, and is consistent with the speed of sound in the heated membrane, calculated to be between 4,000 and $6,000 \mathrm{~ms}^{-1}$ 7].

Additionally, phase retrieval techniques can be applied to each individual coherent diffraction snapshot to produce real-space images of the sample at each time step. This provides a convenient real-space representation of the sample at each time step, enabling researchers to directly visualise sample evolution over time. Figure 13 shows real-space reconstructions for the sample studied in [7], where separate diffraction patterns have been indi-

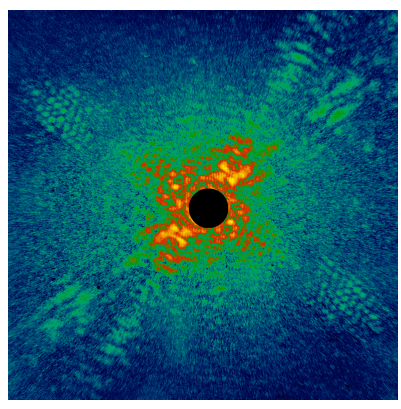

(a) $-5 p s$

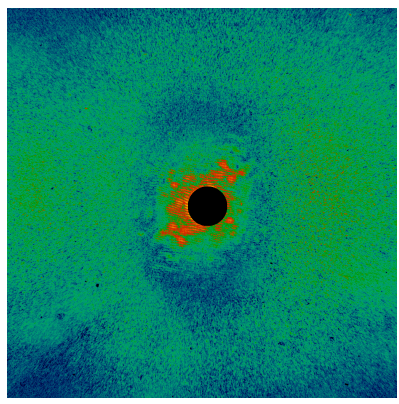

(c) $15 \mathrm{ps}$

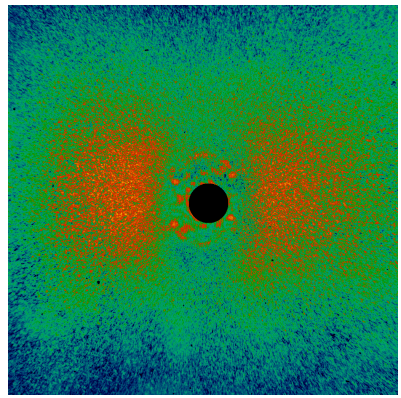

(e) $40 p s$

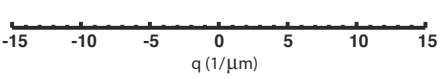

FIG. 11. Time-resolved coherent X-ray scattering: Single shot X-ray diffraction patterns of a silicon membrane driven well beyond equilibrium conditions by a visible light drive laser at time delays of -5 ps (corresponding to the object just before the laser excitation pulse) and at 10 ps, 15 ps, $20 \mathrm{ps}, 40 \mathrm{ps}$, and $140 \mathrm{ps}$ after the laser pulse. The increase in speckle intensity at lower frequencies at longer delays combined with loss of high-spatial frequency information quantify the loss of spatial order as the sample ablates and turns into a plasma. Various analysis techniques including time-resolved small angle X-ray scattering (SAXS) and time-resolved photon correlation spectroscopy (XPCS) can be applied to this type of data. Figure extracted from Barty et.al. 2008

vidually reconstructed using established iterative phase retrieval techniques [16, 48]. These images confirm that the object has changed little in the first $10 \mathrm{ps,} \mathrm{with}$ the edges of the etched structure appearing only slightly blurred. However, by $15 \mathrm{ps}$ disintegration of the membrane itself is clearly visible and only a portion of the 


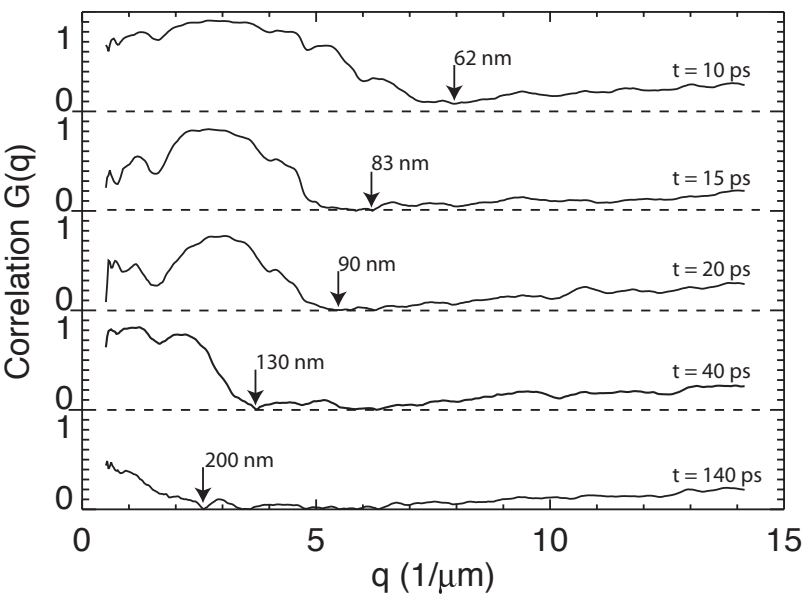

FIG. 12. Measuring the loss of sample order through cross-correlation: Resolution-dependent photon correlation spectra between the delayed diffraction patterns with the original object, showing the progressive loss of mesoscale order in the film structure information as a function of time. Arrows indicate the largest linewidth at which the correlation function first approaches zero, quantitatively estimating the length scale over which order is lost in the sample. Figure extracted from Barty et.al. 2008

pattern is recognizable. This is once again consistent with the expansion of surfaces as the speed of sound in which case the $130 \mathrm{~nm}$ lines and dots of the etched pattern should close up $10-15$ ps after the excitation pulse, and the reference dot can be seen to be half closed at $t=10 \mathrm{ps}$ and no longer visible (corresponding to fully closed over)at $\mathrm{t}=15 \mathrm{ps}$.

Single-shot diffraction imaging as demonstrated in this experiment is unique in its ability to image phenomena where radiation-induced damage and intrinsic sample movement prevent the repeated accumulation of high-resolution scattering data using X-ray sources with longer, weaker pulses. By combining this technique with the shortest available FEL wavelengths [1 and proven synchronization methods [14 this technique could be straightforwardly extended to sub-nm spatial and sub100 fs temporal resolution.

\section{PERSPECTIVES ON THE FUTURE: LCLS, XFEL AND BEYOND}

The future prospects for time resolved imaging at upcoming XFEL sources is extremely bright and the coming years promise to be an extremely exciting time in ultrafast science. The Linac Coherent Light Source (LCLS) in Stanford, CA [12, 40, has just become available to researchers within the last year, and both the European XFEL 23] and Japanese Spring8 Compact Sase Source (SCSS) 63] will very soon come become available to researchers. Further FEL facilities around the world are

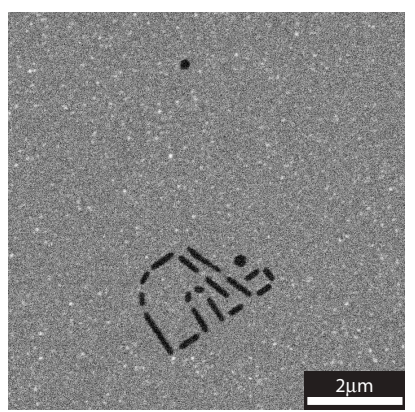

(a) SEM image

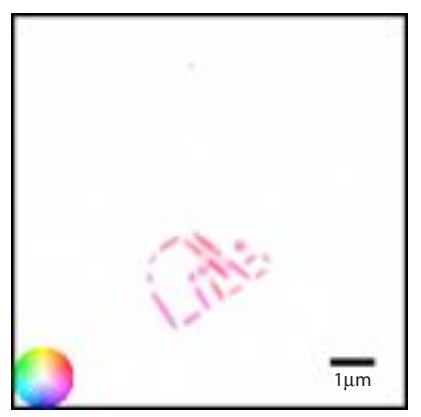

(c) $10 \mathrm{ps}$

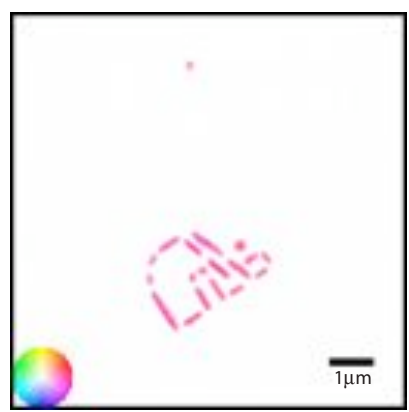

(b) $-5 p s$

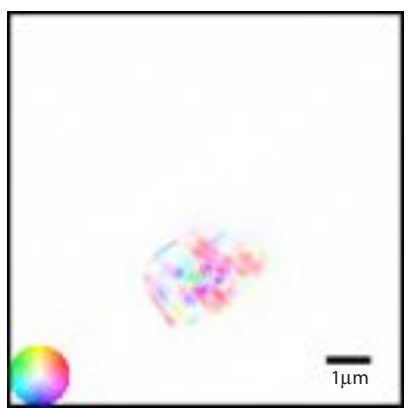

(d) $15 \mathrm{ps}$
FIG. 13. Imaging sample evolution: (a) Scanning electron microscopy (SEM) image of the nano reference object used in the experiment. (b)(d) Real-space images of the sample evolution at various time delays after the laser excitation pulse obtained using iterative phase retrieval techniques: (b) object before the laser excitation pulse, then (c) at 10 ps delay and (d) 15 ps delay. The reconstructed complex-valued object is represented by hue and saturation (as indicated in the colour wheel). Figure extracted from Barty et.al. 2008

either planned, under construction, or in the proposal phase. Meanwhile the first-ever X-ray FEL, FLASH, is in the process of upgrades that will see increases in the photon energy, power and coherence of the X-rays produced 25].

A key feature of these new sources is the availability of higher energy X-rays (ie: shorter wavelengths) - up to $12 \mathrm{keV}$ (1 Åwavelength) - which in turn increases both the penetrating power and pushes the achievable spatial resolution in diffraction experiments below $1 \mathrm{~nm}$ towards atomic resolution. Meanwhile the X-ray pulse length continues to decrease - down to 5 fs in some cases - resulting in higher temporal resolution and a faster shutter speed capable of freezing atomic motion and even approaching the timescales of fundamental atomic processes such as Auger decay. At the same time the peak power and number of photons per pulse continues to rise even as the photon energy is increased.

The implications of these new time-resolved imaging capabilities are truly wide-ranging. The recovery of three-dimensional electron maps of isolated molecules may be possible using XFEL sources if pulses can be produced have sufficiently high intensity that diffraction patterns can be collected in a single pulse, whilst simul- 




B

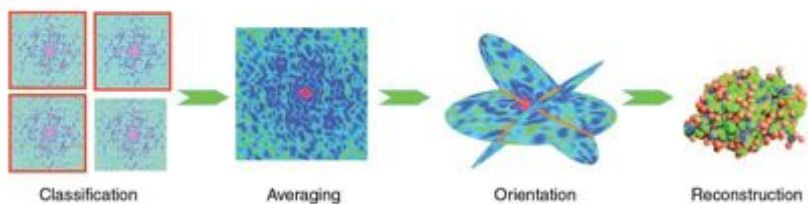

FIG. 14. Biological imaging beyond conventional radiation damage limits: A stream of molecules is injected into the FEL beam. The FEL pulse intensity is sufficiently high that diffraction patterns can be collected in a single pulse, whilst the pulses are short enough to outrun the inevitable radiation damage processes. Individual diffraction patterns from many random particle orientations are computationally summed in three dimensions to yield a three-dimensional diffraction volume. The use of phase retrieval techniques on this diffraction volume leading to recovery of the electron density map. This form of imaging will only possible if the X-ray pulses are sufficiently short to beat the inevitable radiation damage effects causing the molecules to disintegrate. Figure extracted from Gaffney et.al. 2007.

taneously being short enough to outrun the inevitable radiation damage processes. With potentially revolutionary application in structural biology, enabling the structural determination of virtually any macromolecule or virus, understanding of the time-resolved response of molecules to the extreme conditions produced by XFEL pulses is critical to determining the practical resolution limits achievable using this technique.

In time resolved biological studies XFEL sources may ultimately enable the imaging of biochemical reactions as they progress. For example, studies of the COphotodetachment in crystalline myoglobin [61] have been limited by the picosecond pulse lengths available at synchrotrons. XFEL sources enable this time resolution to be pushed into the femtosecond regime, and such experiments will happen soon at LCLS. In the future it may be possible to study such reactions including the photoreaction of bacteriorhodopsin and possibly even the process of protein folding on sub-picosecond time scales, revealing biology in action and leading to greater understanding of how biological machines function.
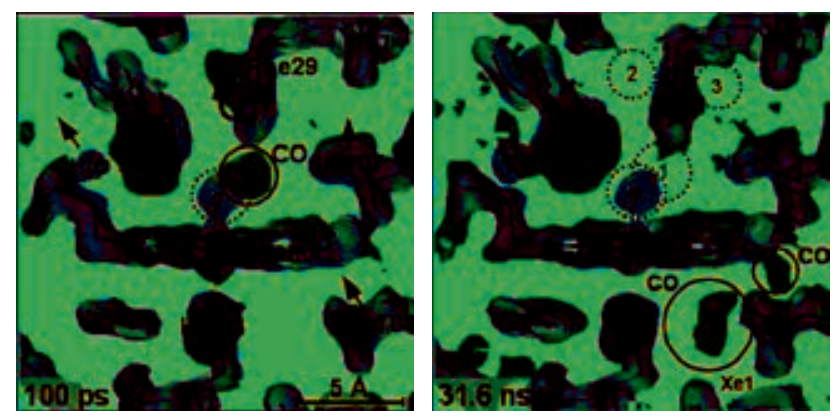

FIG. 15. Time-resolved bioimaging: Time-resolved structure of the heme region of myoglobin: $\mathrm{CO}$, showing the photodetachment of $\mathrm{CO}$, its migration to sites 2 and 3, and the relaxation re- sponse of the neighboring protein structures Figure extracted from Schotte et.al. 2003.

Meanwhile, in materials science time-resolved X-ray diffraction will enable the behaviour of transient states such as grain boundary formation, dislocation propagation and crystalline phase transitions as they occur. For example, large scale molecular dynamics simulations by Kadau et.al. 39] predict crystalline phase transitions in metals induced by the passage of a shock wave. Now, using FEL sources it should be possible to image such transitions as they occur in time-resolved diffraction experiments. This will lead to new insights into how materials evolve with time and enabling the design of engineered materials with new properties for a range of new applications. In this regard the superior beam quality available from XFEL sources provides significantly better data than available from current sources, whilst the the high spatial coherence enables the use of coherent scattering and coherent imaging techniques to reveal sample evolution. With the progress of XFEL sources towards shorter wavelengths and higher pulse power it will soon be possible to perform experiments at the same spatial and temporal timescales as state-of-the-art molecular dynamics simulation. Furthermore techniques such as near-edge X-ray absorption and fine structure microscopy techniques can be used to provide chemical specific contrast in time resolved imaging experiments [2].

What is clear is that by combining single shot coherent imaging with the femtosecond X-ray pulses produced by free electron lasers it is now possible to truly freeze the sample motion on femtosecond to picosecond timescales with simultaneous Ångstrom to nanometre spatial resolution. The list of new applications for XFEL sources goes on as amply documented in the science cases for new facilities being proposed worldwide. While experimental investigations combining this range of spatial and temporal scales were unimaginable just a decade ago, the advent of X-ray free electron lasers have brought these goals within reach for the first time. The equivalent advancements in simulation capabilities now mean that simulations and experiments can be performed at overlapping temporal and spatial scales. 
The advent of X-ray free electron lasers such as FLASH have already opened up new new frontiers in time resolved science, and will continue to do so as new sources become available at higher X-ray energies, with shorter pulses and with higher peak powers. We are about to witness a transition from the investigation of static sys- tems to time-resolved studies of the dynamics of physical, chemical and biochemical processes on near-atomic length scales at their natural timescales of femtoseconds, and along with this transition will come the knowledge needed to shape and control matter at the most fundamental levels.
[1] Ackerman, W. et.al. Operation of a free-electron laser from the extreme ultraviolet to the water window. Nature Photonics 1, 336 (2007)

[2] Ade, H. and Stoll, H. Near-edge X-ray absorption finestructure microscopy of organic and magnetic materials, Nature Materials 8, 281 (2009)

[3] Armstrong, M.R. et.al. Practical considerations for high spatial and temporal resolution dynamic transmission electron microscopy. Ultramicroscopy 107, 356 (2007)

[4] V. Ayvazyan et.al., First operation of a free-electron laser generating GW power radiation at $32 \mathrm{~nm}$ wavelength. Eur. Phys. J. D 37, 297-303 (2006).

[5] Bajt, S. et.al. A camera for coherent diffractive imaging and holography with a soft-X-ray free electron laser. Applied Optics 47, 1673 (2008).

[6] Bartels, R.A. et.al. Generation of spatially coherent light at extreme ultraviolet wavelengths Science 297, 376 (2002)

[7] Barty, A. et.al. Ultrafast single-shot diffraction imaging of nanoscale dynamics Nature Photonics 2, 415 (2008)

[8] Bergh, M., Timneanu, N. O. and van der Spoel, D. Model for the dynamics of a water cluster in an x-ray free electron laser beam. Phys. Rev. E 70, 051904 (2004).

[9] Bogan, M.J. et.al Aerosol Imaging with a Soft X-Ray Free Electron Laser, Aerosol Science and Technology, 44, ivi (2010)

[10] Bogan, M.J. Imaging aerosol particles, J. Phys. B. Need reference from current JPhysB issue (2010)

[11] Born, E. and Wolf, E. Principles of Optics

[12] Boutet, S. and Williams, G.J., The Coherent X-ray Imaging (CXI) instrument at the Linac Coherent Light Source (LCLS), New Journal of Physics 12, 035024 (2010).

[13] Brauer, S. et.al. X-ray intensity fluctuation spectroscopy observations of critical dynamics in $\mathrm{Fe}_{3} \mathrm{Al}$. Phys.Rev.Lett. 74, 2010 (1995)

[14] Cavalieri, A.L. et.al. Clocking Femtosecond X Rays. Phys.Rev.Lett 94, 114801 (2005)

[15] Chao, W. et.al, Soft X-ray microscopy at a spatial resolution better than $15 \mathrm{~nm}$, Nature 435, 1210 (2005).

[16] Chapman, H.N. et.al. Femtosecond Diffractive Imaging with a Soft-X-ray Free-Electron Laser. (2006) Nature Physics 2, 839

[17] Chapman, H.N. et.al. High-resolution ab initio threedimensional X-ray diffraction microscopy. J. Opt. Soc. Am. A 23, 1179-1200 (2006).

[18] Chapman, H.N. et.al. Femtosecond time-delay X-ray holography. Nature 448, 676 (2007).

[19] Chapman, H.N. X-ray imaging beyond the limits, Nature Materials 8, 299 (2009)

[20] Dierker, Pindak, S.R., Fleming, R., Robinson, I. \& Berman, L. X-ray photon correlation spectroscopy study of Brownian motion if gold colloids in glycerol. Phys.Rev.Lett. 75, 449 (1995)
[21] Ding, Y. et.al. Measurements and Simulations of Ultralow Emittance and Ultrashort Electron Beams in the Linac Coherent Light Source Phys.Rev.Lett. 102, 254801 (2009)

[22] Elser, V. Phase retrieval by iterated projections J.Opt.Soc.Am.A. 20, 40 (2003)

[23] http://xfel.eu

[24] Fienup, J.R. Reconstruction of an object from the modulus of its Fourier transform Opt.Lett. 3, 27 (1978)

[25] http://flash.desy.de

[26] Fritz, D.M. et al. Ultrafast Bond Softening in Bismuth: Mapping a Solid's Interatomic Potential with X-rays, Science 315, 633 (2007).

[27] Gaffney, K.J. et al. Observation of Structural Anisotropy and the Onset of Liquid-like Motion during the Nonthermal Melting of InSb, Phys. Rev. Lett. 95, 125701 (2005).

[28] Gaur, U. and Wunderlich, B. Heat capacity and other thermodynamic properties of linear macromolecules. V. Polystyrene. J. Phys. Chem. Ref. Data 11, 313325 (1982).

[29] Gutt, C.,Leupold, O. \& G. Grübel, G. Surface XPCS on nanometer length scales - what can we expect from an X-ray free electron laser? Thin Solid Films 515, 5532 (2007)

[30] Grübel, G., Stephenson, G.B., Gutt, C., Sinn, H. \& Tschentscher, T. XPCS at the European X-ray free electron laser facility Nucl.Instr.Meth.B 267, 357 (2007)

[31] Hau-Riege, S.P. et.al. SPEDEN: reconstructing single particles from their diffraction patterns Acta.Cryst.A. 60, 294 (2004)

[32] Hau-Riege, S.P., London, R.A., Chapman, H.N., \& Bergh, M. Soft-x-ray free-electron-laser interaction with materials. Phys Rev E 76, 046403 (2007).

[33] S.P.Hau-Riege et.al. Sacrificial Tamper Slows Down Sample Explosion in FLASH Diffraction Experiments Phys.Rev.Lett 104, 064801 (2010)

[34] Henderson, R. The potential and limitations of neutrons, electrons and X-rays for atomic resolution microscopy of unstained biological molecules. Quart. Rev. Biophys. 28, 171193 (1995).

[35] Howells, M. R. et al. An assessment of the resolution limitation due to radiation-damage in x-ray diffraction microscopy. J. Electron. Spectrosc. Relat. Phenom.170, 4-12 (2009).

[36] Huang, X. et.al Soft X-Ray Diffraction Microscopy of a Frozen Hydrated Yeast Cell, PhysRev.Lett. 103, 198101 (2009).

[37] Ischebeck, I et.al Study of the transverse coherence at the TTF free electron laser. Nucl. Instr. and Meth. A 507, 175-180 (2003)

[38] Jurek, Z., Faigel, G. and Tegze, M. Dynamics in a cluster under the influence of intense femtosecond hard x-ray pulses. Eur. Phys. J. D 29, 217229 (2004). 
[39] Kadau, K. /emphet.al. Microscopic view of structural phase transitions induced by shock waves, Science 296, 1681 (2002)

[40] http://lcls.slac.stanford.edu/

[41] Lee, R. et al. Finite temperature dense matter studies on next-generation light sources. J. Opt. Soc. Am. B 20, 770778 (2003).

[42] Lima, E. et.al. Cryogenic X-Ray Diffraction Microscopy for Biological Samples, Phys.Rev.Lett 103, 198102 (2009).

[43] Lindenberg, A.M. et al. Atomic-Scale Visualization of Inertial Dynamics, Science 308, 392 (2005).

[44] Lindenberg, A.M. et.al X-Ray diffuse scattering measurements of nucleation dynamics at femtosecond resolution ,Phys. Rev. Lett. 100, 135502 (2008).

[45] Lorazo, P. et.al Thermodynamic pathways to melting, ablation, and solidification in absorbing solids under pulsed laser irradiation, Phys Rev B. 73, 134108 (2006)

[46] Luke, D.R., Relaxed averaged alternating reflections for diffraction imaging. Inverse Problems 21, 37-50 (2005).

[47] Mancuso, A.P. et.al Coherent imaging of biological samples with femtosecond pulses at the free-electron laser FLASH, New Journal of Physics 12, 035003 (2010)

[48] Marchesini, S. et.al. X-ray image reconstruction from a diffraction pattern alone. Phys. Rev. B 68, 140101 (2003).

[49] Marchesini, S. A unified evaluation of iterative projection algorithms for phase retrieval, Rev.Sci.Instrum 78, 011301 (2007)

[50] Marchesini, S. et.al Massively parallel X-ray holography, Nature Photonics, 2, 560 (2008)

[51] Miao, J., Sayre, D. and Chapman, H.N. Phase retrieval from the magnitude of the Fourier transforms of nonperiodic objects, J. Opt. Soc. Am. A 15, 1622 (1998).

[52] Miao, J., Charalambous, P., Kirz, J. \& Sayre, D. Extending the methodology of X-ray crystallography to allow imaging of micrometre-sized non-crystalline specimens Nature, 400, 42 (1999)

[53] More, R.M., Warren, K.H., Young, D.A., \& Zimmerman, G.B. A new quotidian equation of state (QEOS) for hot dense matter. Phys. Fluids 31, 3059 (1988) .

[54] Neutze, R. et.al. Potential for biomolecular imaging with femtosecond X-ray pulses. Nature 406, 752757 (2000).

[55] K.A. Nugent, Coherent Methods in the X-ray sciences, Advances in Physics, 59, 1 (2010).

[56] Radcliffe, P. et al. An experiment for two-color photoionization using high intensity extreme-UV free electron and near-IR laser pulses. Nuclear Instruments and Methods A583, 516-525 (2007)

[57] Rischel, C. et.al. Femtosecond time-resolved X-ray diffraction from laser-heated organic films. Nature 390, 490 (1997).

[58] Rose-Petruck, C. et.al. Picosecond milliÅngström lattice dynamics measured by ultrafast X-ray diffraction Nature 398, 310 (1999).

[59] Saldin, E.L., Schneidmiller, E.A., \& Yurkov, M. The Physics of Free-Electron Lasers, Springer, Berlin, 2000.

[60] Sayre, D. Some implications of a theorem due to Shannon, Acta Cryst. 5, 843 (1952)

[61] Schotte, F., et al., Watching a protein as it functions with 150-ps time-resolved X-ray crystallography, Science 300, 1944 (2003).

[62] Schoenlein, R.W. et.al. Generation of Femtosecond Pulses of Synchrotron Radiation Science 287, 2237 (2000)

[63] http://www-xfel.spring8.or.jp
[64] Shapiro, D. et.al. Biological imaging by soft x-ray diffraction microscopy, PNAS 102, 15343 (2005).

[65] Siders, C.W. et.al. Direct Measurement of Non-Thermal Melting using Ultrafast X-ray diffraction. Science 286, 1340, (1999).

[66] Sokolowski-Tinten, K. et.al Femtosecond X-ray measurement of coherent lattice vibrations near the Lindemann stability limit. Nature 422, 287-289 (2003).

[67] Sokolowski-Tinten, K. et.al. Transients states of matter during short pulse laser ablation. Phys. Rev.Lett. 81, 224, (1998).

[68] Sokolowski-Tinten, K. et.al. Femtosecond X-ray measurement of coherent lattice vibrations near the Lindemann stability limit. Nature 422, 287 (2003).

[69] Solem, J. C. and Baldwin, G. C. Microholography of living organisms. Science 218, 229235 (1982).

[70] Upadhyay, A.K. et.al Ablation by ultrashort laser pulses: Atomistic and thermodynamic analysis of the processes at the ablation threshold Phys Rev. B. 78, 045437 (2008)

[71] Vartaniants, I. Coherent X-ray Imaging, J. Phys. B. Need reference from current JPhysB issue (2010)

[72] Will, I, Koss, G. \& Templin, I. The upgraded photocathode laser of the TESLA Test Facility. Nucl. Instr. and Meth. A541, 467-477 (2005)

[73] Young, J.F., Preston, J.S., van Driel, H.M. \& Sipe, J.E. Laser-induced periodic surface structure. II. Experiments on Ge, Si, Al, and brass. Phys. Rev. B. 27, 1155 (1983)

[74] Lobastov, V.A., Srinivasan, R., \& Zewail, A.H. Four-dimensional ultrafast electron microscopy. Proc.Natl.Acad.Sci.USA. 102, 7069 (2005).

[75] Zhakhovskii, V.V. et.al Molecular-dynamics simulation of rarefaction waves in media that can undergo phase transitions, JETP Lett. 71, 167 (2000) 